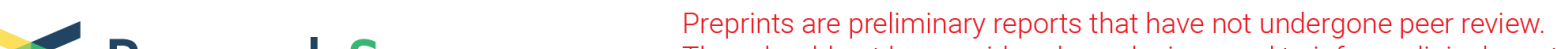 Research Square
They should not be considideded onnlusive used to inform clinical practice,
or referenced by the medias validated information.
}

\section{Extra \& Intravascular Ultrasound (E\&IVUS) Guided Intervention for Femoropopliteal Arterial Occlusive Lesions.}

Takuya Haraguchi ( $\nabla$ t.haraguchi@scvc.jp )

Sapporo Heart Center https://orcid.org/0000-0002-2116-2178

Nozomi Sawada

Sapporo Heart Center

Masanaga Tsujimoto

Sapporo Heart Center

Masato Furuya

Sapporo Heart Center

Saori Itai

Sapporo Heart Center

Tsuyoshi Takeuchi

Sapporo Heart Center

Yoshifumi Kashima

Sapporo Heart Center

Katsuhiko Sato

Sapporo Heart Center

Tsutomu Fujita

Sapporo Heart Center

\section{Research Article}

Keywords: Superficial femoral artery, Extravascular ultrasound, Intravascular ultrasound, Chronic total occlusion, Endovascular therapy, Peripheral arterial disease

Posted Date: November 3rd, 2021

DOI: https://doi.org/10.21203/rs.3.rs-1009706/v1

License: (c) (1) This work is licensed under a Creative Commons Attribution 4.0 International License.

Read Full License 


\section{Abstract}

Background: Intravascular ultrasound (IVUS) shows vascular structures and positions of interventional devices in cross sectional-short axis to support interventions, especially for complex lesions. On the other hand, extravascular ultrasound (EVUS) visualizes the devices and vessel structures in long and short axis and reduces the radiation exposure by avoiding the use of fluoroscopy during guidewire manipulation. The images obtained from EVUS handling to guide the guidewire manipulation in both long and short axis is more difficult, time consuming, and stressful than IVUS, which is in short axis only. To solve this issue, we propose a novel guidewire crossing method in conjunctive with the use of both modalities, named "extra and intravascular ultrasound (E\&IVUS)" guided intervention.

Main text: This is the first report of a combined use of EVUS and IVUS for femoropopliteal occlusions. EVUS-guided intervention is mandatory to check the position of the device in long and short axis. However, the images of long axis are sometimes different from the original ones when EVUS is required to image from the short axis to the long axis. E\&IVUS allows to dedicate EVUS to acquisition of the long axis and IVUS to the short axis view when confirming the device position. As a result, E\&IVUS shortens the operation time and reduces the stress due to the manipulation of the probe to switch from the long to short axis image and vice versa. Moreover, we can accurately manipulate the guidewire to perform intimal tracking according to the information of EVUS long axis and the IVUS short axis images. Case involved a 76-year-old female with right superficial femoral artery occlusion was angiographically contrasted from a contralateral 6-Fr sheath. A hard wire supported with an over-the-wire typed IVUS was advanced into the CTO with EVUS and IVUS to confirm their positions until the guidewire crossing. We repeatedly performed this process, and all intimal tracking succeeded. The drug-coating balloons appropriately sized by IVUS measurement were deployed. Finally, a sufficient blood flow was achieved without complications.

Conclusions: E\&IVUS is a preferred strategy than using EVUS or IVUS alone. We should evaluate the clinical outcomes of this technique.

\section{Background}

Intravascular ultrasound (IVUS) shows vascular structures and positions of interventional devices in cross sectional-short axis to support interventions, especially for complex morphological lesions. On the other hand, extravascular ultrasound (EVUS) is a useful diagnostic device and a new therapeutic assistive device for guiding and monitoring the progress of peripheral interventions (Katzenschlager. 1996). EVUS visualizes the devices and vessel structures in long and short axis and reduces the radiation exposure by avoiding the use of fluoroscopy during guidewire manipulation. The efficacy of IVUS-guided or EVUSguided intervention for chronic total occlusion (CTO) has been reported (lida. 2014, Mustapha. 2014). Both methods facilitate the intraplaque tracking to yield better outcomes (Mori. 2021, Tsubakimoto. 2021). 
However, there is a learning curve to demonstrate and appreciate the structures imaged with both imaging modalities. EVUS handling to present the proper images for guidewire manipulation in both long and short axis is more difficult, time consuming, and stress between an operator and a sonographer than IVUS in only short axis. Therefore, EVUS is a useful device but not yet widely used. To solve this issue, we propose a novel guidewire crossing method in conjunctive with the use of both modalities, named "extra and intravascular ultrasound (E\&IVUS)" guided intervention.

\section{Main Text}

This is the first report of a combined use of EVUS and IVUS for femoropopliteal CTOs. EVUS-guided intervention is mandatory to check the position of the device in long and short axis during CTO intervention. However, this maneuver consumes the operation time. The long axis direction is sometimes different from the original long axis direction when EVUS is handled to image from the short axis to the long axis. E\&IVUS allows to dedicate EVUS to the long axis and IVUS to the short axis view when confirming the device position. As a result, E\&IVUS shortens the operation time and reduce the stress due to the manipulation of the probe to show the long and short axis image. Moreover, we can accurately manipulate the guidewire to perform intimal tracking according to the information of EVUS long axis and the IVUS short axis images.

The process of E\&IVUS is as follows. A 6-Fr guiding sheath as an ipsilateral or a crossover approach is inserted, and unfractionated heparin (5000-IU) is injected intra-arterially. A sonographer stands on the opposite side of the operator. EPIQ Elite (Philips, Netherlands) with $22 \mathrm{MHz}$ linear probes is used to identify the long axis image during guidewire manipulation. An over-the-wire (OTW) type of IVUS (Visions PV.018 OTW, Philips) is used to not only visualize and analyze the lesions in short axis but support the guidewire force. During the E\&IVUS procedure, a C-arm is moved away from the operative field to avoid radiation exposure. A hard 0.018-in. guidewire (Astato, Asahi Intec., Japan) is manipulated through the IVUS-OTW lumen to advance into the occlusions. The probe must be fixed in position of showing the center of the vessel, and the operator should gently manipulate and advance the guidewire with maintaining clear delineation of the guidewire in the EVUS long axis image (Fig. 1). We use needle visualization mode of EPIQ Elite. In this mode, when the guidewire tip and the echo beam were overlapped in the long axis direction, echo reflection occurs and the tip of the guidewire glows (Fig. 2a). By advancing the wire without deviating from the EVUS long axis, the wire can be advanced into a plaque in the proper direction. Therefore, there is no need to confirm the tip of the guidewire in the EVUS short axis. Also, we divided the EVUS long axis image of the occlusion into seven sections and gave each one a name (Fig. 2b). After the guidewire is advanced from P1 to D3 segment, IVUS follows it with confirming the position of the guidewire, plaque quality, and vessel size in the IVUS short axis image. This process is repeated until the wire crosses the occlusion. After guidewire crossing, IVUS passes through the lesion following the guidewire, and we replace it with a soft guidewire through the IVUS-OTW lumen. Then, IVUS images are collected by manual pullback through the occlusions. Finally, the lesions are revascularized with a balloon and/or scaffolds appropriately sized from IVUS analysis. 
Here, we present the representative case of treatment with the E\&IVUS guided intervention. A 76-year-old female with severe claudication with $\mathrm{ABI}$ of 0.58 due to right superficial femoral artery (SFA) occlusion of 24-cm was angiographically contrasted from a contralateral 6-Fr sheath (Fig. 3a). Astato supported with Visions PV.018 OTW was advanced to the CTO orifice, and EVUS confirmed their positions in long axis after C-arm was moved away. We gently manipulated the guidewire to advance into the CTO with EVUS guidance. The guidewire reached the D3 segment in EVUS long axis, then, IVUS followed it and confirmed that the guidewire route was in the intima and assessed the plaque quality and vessel size. We repeatedly performed this process, and all intimal tracking through the entire occlusion succeeded (Fig. 3b). The drug-coating balloon appropriately sized by IVUS measurement during E\&IVUS procedure were deployed. Finally, a sufficient lumen area and blood flow was achieved without complications (Fig. 3c, d). We have not observed periprocedural complications, restenosis, reinterventions, and limb loss a year after the procedure.

In terms of limitations of E\&IVUS are here. First, IVUS resonates the frequency of EVUS, and it causes the noises in the IVUS image. Adjusting the depth of IVUS image can eliminate the effects caused by the noise. Second, parallel wire technique is almost impossible with 6-Fr sheath due to Visions PV.018 OTW of $5 \mathrm{Fr}$. If we perform this technique, we should use the 7-Fr sheath or another thinner type of IVUS. Final, calcified vessels and the distal SFA segment below the adductor canal are poorly visualized with EVUS. We should recognize the limitations of EVUS and change the treatment strategy.

\section{Conclusions}

E\&IVUS is a preferred strategy than using EVUS or IVUS alone. We should evaluate the clinical outcomes of this technique.

\section{List Of Abbreviations}

Intravascular ultrasound (IVUS)

Extravascular ultrasound (EVUS)

Chronic total occlusion (CTO)

Extra and Intravascular ultrasound (E\&IVUS)

Over-the-wire (OTW)

superficial femoral artery (SFA)

\section{Declarations}

\section{Ethics approval and consent to participate}


Informed consent was achieved. The report followed the declaration of Helsinki of 1964.

\section{Consent for publication}

Written informed consent was obtained from the patient for publication of this case report and any accompanying images.

\section{Availability of data and material}

The datasets used and/or analyzed during the current study are available from the corresponding author on reasonable request.

\section{Competing interests}

The authors declare that they have no competing interests.

\section{Funding}

None

\section{Authors' contributions}

All authors contributed to the study conception and design. The first draft of the manuscript was written by Takuya Haraguchi and all authors commented on previous versions of the manuscript. All authors read and approved the final manuscript.

\section{Acknowledgements}

We thank for Tanimura T., Miyoshi T., as special team member in PAD Center.

\section{References}

1. Katzenschlager R, Ahmadi A, Minar E, Koppensteiner R, Maca T, Pikesch K, Stümpflen A, Ugurluoglu A, Ehringer $H$ (1996) Femoropopliteal artery: initial and 6-month results of color duplex US-guided percutaneous transluminal angioplasty. Radiology 199(2):331-4. DOI:

10.1148/radiology.199.2.8668773

2. lida O, Takahara M, Soga Y, Suzuki K, Hirano K, Kawasaki D, Shintani Y, Suematsu N, Yamaoka T, Nanto S, Uematsu M (2014) Efficacy of intravascular ultrasound in femoropopliteal stenting for peripheral artery disease with TASC II class A to C lesions. J Endovasc Ther 21(4):485-492. DOI: 10.1583/14-4721R.1

3. Mustapha JA, Saab F, McGoff T, Heaney C, Diaz-Sandoval L, Sevensma M, Karenko B (2014) Tibiopedal arterial minimally invasive retrograde revascularization in patients with advanced peripheral vascular disease: the TAMI technique, original case series. Catheter Cardiovasc Interv 83(6):987994. DOI: $10.1002 / \operatorname{ccd} .25227$ 
4. Mori S, Hirano K, Yamawaki M, Kobayashi N, Sakamoto Y, Tsutsumi M, Honda Y, Makino K, Shirai S, Ito $Y$ (2021) Usefulness of ultrasound-guided intraluminal approach for long occlusive

femoropopliteal lesion. Heart Vessels 36(3):376-382. DOI: 10.1007/s00380-020-01697-8

5. Tsubakimoto Y, Isodono K, Fujimoto T, Kirii Y, Shiraga A, Kasahara T, Ariyoshi M, Irie D, Sakatani T, Matsuo A, Inoue K, Fujita H (2021) IVUS-Guided Wiring Improves the Clinical Outcomes of Angioplasty for Long Femoropopliteal CTO Compared with the Conventional Intraluminal Approach. J Atheroscler Thromb 28(4):365-374. DOI: 10.5551/jat.57166

\section{Figures}

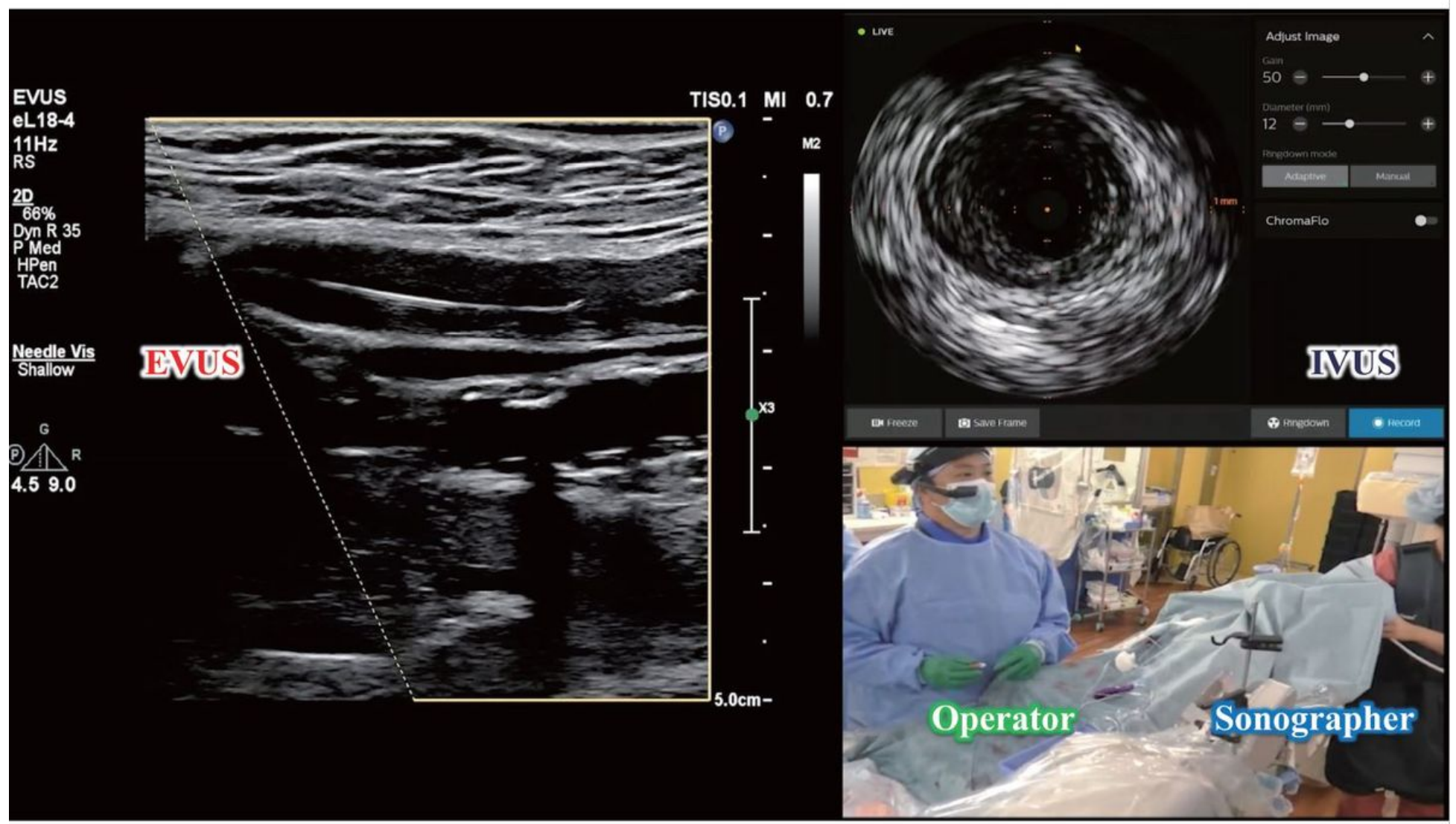

Figure 1

The representative image of E\&IVUS-guided intervention. 

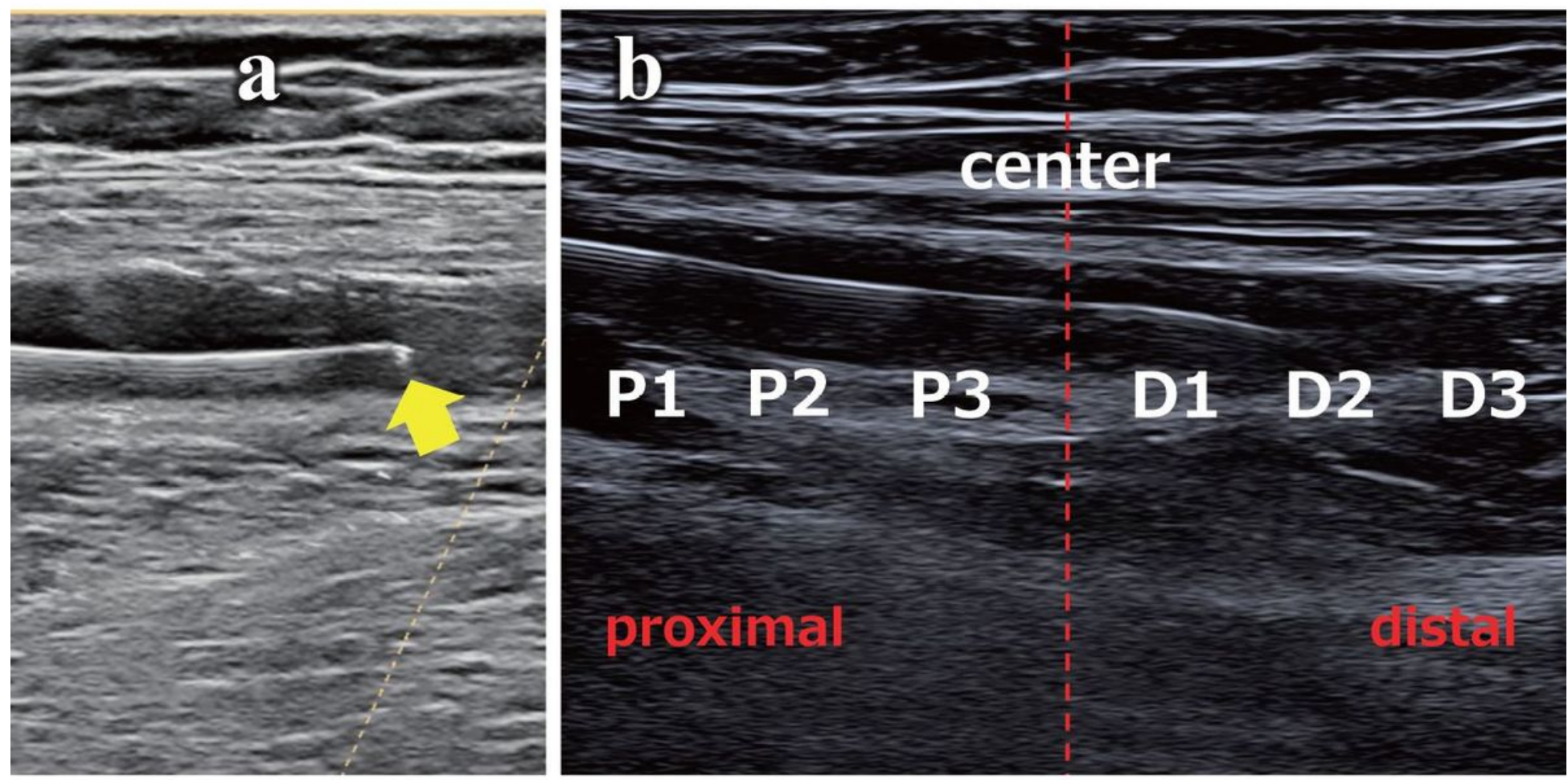

Figure 2

The representative image of EVUS. a. A tip of a guidewire is reflected by EVUS beam, especially with needle visualization mode of EPIQ Elite (Philips, Netherlands), when both directions are overlapped (yellow arrow). The guidewire can be advanced into the relative center of the lesions if the wire is advanced while maintaining this reflex of the tip of the guidewire. b. The EVUS image in long axis is divided into seven sections to create a mutual understanding with an operator and a sonographer. 

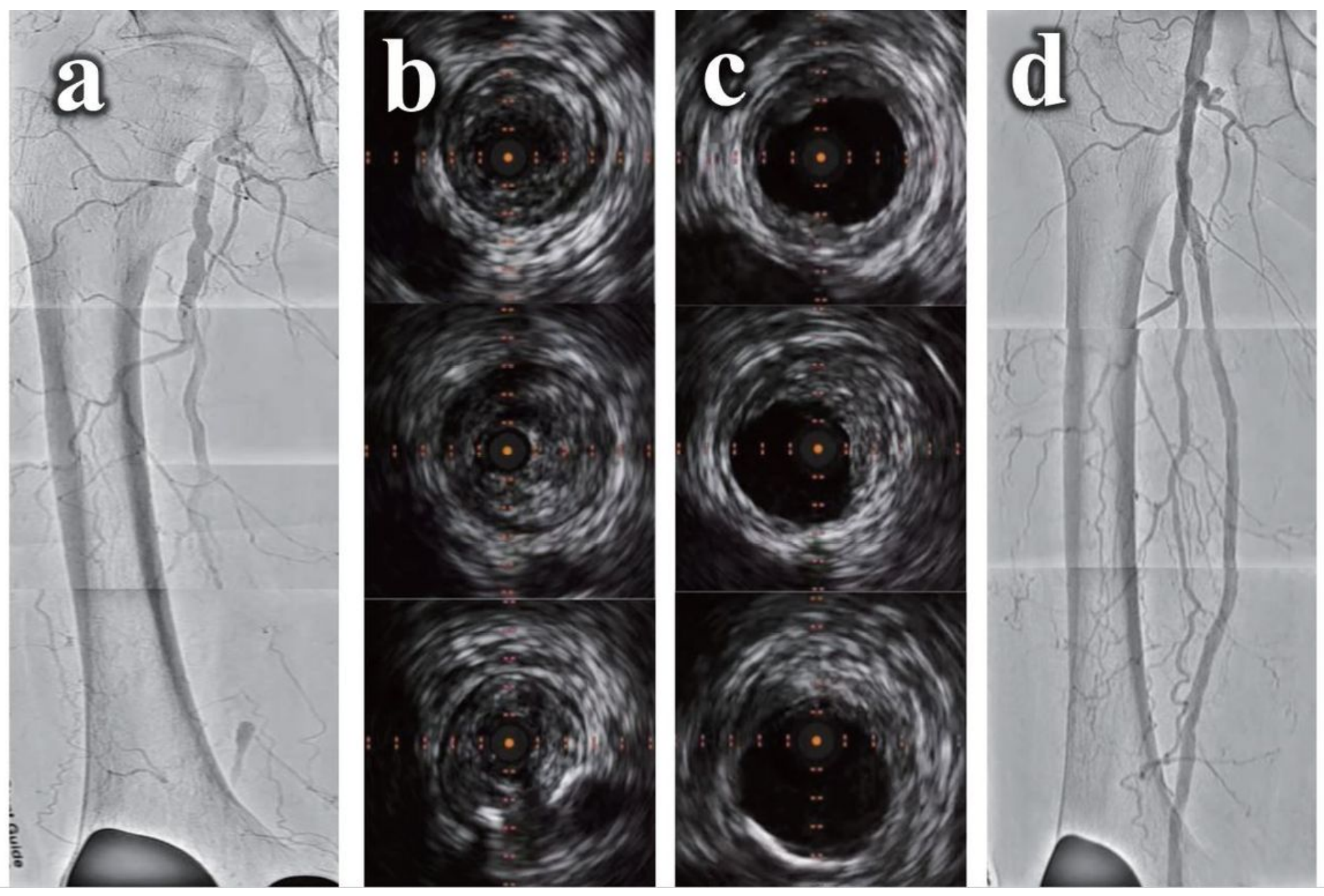

\section{Figure 3}

The representative case of E\&IVUS-guided intervention. a. Control angiography of right superficial femoral arterial occlusion. b. IVUS images of all intimal tracking with E\&IVUS procedure. c. IVUS images of a sufficient minimal lumen area after drug-coating balloon dilatation. d. Final angiography after E\&IVUSguided intervention for superficial femoral arterial occlusion. 